\title{
Correction to: Risk factors and impact of conversion from VATS to open lobectomy: analysis from a national database
}

\author{
Stefano Bongiolatti ${ }^{1}$ (D) Alessandro Gonfiotti ${ }^{1}$. Domenico Viggiano ${ }^{1} \cdot$ Sara Borgianni ${ }^{1} \cdot$ Leonardo Politi $^{1}$. \\ Roberto Crisci ${ }^{2}$. Carlo Curcio ${ }^{3}$ - Luca Voltolini ${ }^{1} \cdot$ Italian VATS Group
}

Published online: 4 June 2019

○) Springer Science+Business Media, LLC, part of Springer Nature 2019

\section{Correction to: Surgical Endoscopy https://doi.org/10.1007/s00464-019-06682-5}

In the "Results" section of the Abstract, the sentence:

"The mortality rate was similar, but the percentage of patients who suffered from any complication $(41.7 \%$ vs $24.4 \%, \mathrm{p}<0.01)$, the complication rate $(65 \%$ vs $32.2 \%$, $\mathrm{p}<0.01)$, chest tube duration $(\mathrm{p}<0.01)$ and the hospitalisation rate $(\mathrm{p}<0.01)$ were higher for patients converted."

should read:

"The mortality rate was similar, but the percentage of patients who suffered from any complication $(41.7 \%$ vs $24.4 \%, \mathrm{p}<0.01)$, the complication rate $(65 \%$ vs $32.2 \%$, $\mathrm{p}<0.01)$, chest tube duration $(\mathrm{p}<0.01)$ and length of stay $(\mathrm{p}<0.01)$ were higher for patients converted."

Acknowledgements List of collaborators of the Italian VATS Group: Carlo Curcio (Monaldi Hospital, Napoli); Dario Amore (Monaldi Hospital, Napoli); Giuseppe Marulli (University of Padova); Samuele Nicotra (University of Padova); Andrea De Negri (San Martino Hospital, Genova); Paola Maineri (San Martino Hospital, Genova); Gaetano Di Rienzo (Vito Fazzi Hospital, Lecce); Camillo Lopez (Vito Fazzi Hospital, Lecce); Roberto Crisci (University Hospital L'Aquila-Teramo); Duilio Divisi (University Hospital L'Aquila-Teramo); Angelo Morelli (S. Maria delle Misericordia Hospital, Udine); Alessandro Bertani (IRCCS ISMETT-UPMC, Palermo); Emanuele Russo (IRCCS ISMETT-UPMC, Palermo); Francesco Londero (S.

The original article can be found online at https://doi.org/10.1007/ s00464-019-06682-5.

Stefano Bongiolatti

stefanobongiolatti@gmail.com

1 Thoracic Surgery Unit, University Hospital Careggi, Largo Brambilla, 1, 50134 Florence, Italy

2 Department of Thoracic Surgery, University of L'Aquila, L'Aquila, Italy

3 Division of Thoracic Surgery, Monaldi Hospital, Naples, Italy
Maria delle Misericordia Hospital, Udine); Lorenzo Rosso (General Hospital, University of Milan, Milano); Lorenzo Spaggiari (IEO Hospital, Milano); Roberto Gasparri (IEO Hospital, Milano); Guido Baietto (Maggiore della Carita` Hospital, Novara); Caterina Casadio (Maggiore della Carita`Hospital, Novara); Maurizio Infante (Borgo Trento Hospital, Verona); Cristiano Benato (Borgo Trento Hospital, Verona); Marco Alloisio (IRCCS Humanitas, Milano); Edoardo Bottoni (IRCCS Humanitas, Milano); Andrea Droghetti (Carlo Poma Hospital, Mantova); Andrea Giovanardi (Carlo Poma Hospital, Mantova); Giuseppe Cardillo (Forlanini Hospital, Roma); Francesco Carleo (Forlanini Hospital, Roma); Luca Bertolaccini (Maggiore Teaching Hospital, Bologna); Piergiorgio Solli (Maggiore Teaching Hospital, Bologna); Franco Stella (S. Orsola Hospital, Bologna); Giampiero Dolci (S.Orsola Hospital, Bologna); Francesco Puma (University of Perugia); Damiano Vinci (University of Perugia); Giorgio Cavallesco (University of Ferrara); Pio Maniscalco (University of Ferrara); Desideria Argnani (AUSL Romagna Hospital Forli); Luca Ampollini (University of Parma); Paolo Carbognani (University of Parma); Alberto Terzi (Negrar Hospital, Verona); Andrea Viti (Negrar Hospital, Verona); Giampiero Negri (S. Raffaele Hospital, Milano); Alessandro Bandiera (S. Raffaele Hospital, Milano); Reinhold Perkmann (Bolzano Hospital, Bolzano); Francesco Zaraca (Bolzano Hospital, Bolzano); Mohsen Ibrahim (S. Andrea Hospital, Roma); Camilla Poggi (S. Andrea Hospital, Roma); Felice Mucilli (S. Maria Annunziata Hospital, Chieti); Pierpaolo Camplese (S. Maria Annunziata Hospital, Chieti); Luca Luzzi (University of Siena); Marco Ghisalberti (University of Siena); Andrea Imperatori (University of Varese); Nicola Rotolo (University of Varese); Luigi Bortolotti (Humanitas Gavazzeni Hospital, Bergamo); Giovanna Rizzardi (Humanitas Gavazzeni Hospital, Bergamo); Massimo Torre (Niguarda Hospital, Milano); Alessandro Rinaldo (Niguarda Hospital, Milano); Armando Sabbatini (Ospedali Riuniti, Ancona); Majed Refai (Ospedali Riuniti, Ancona); Mauro Roberto Benvenuti (Spedali Civili, Brescia); Diego Benetti (Spedali Civili, Brescia); Alessandro Stefani (Ospedale Policlinico, Modena); Pamela Natali (Ospedale Policlinico, Modena); Paolo Lausi (Ospedale Molinette, Torino); Francesco Guerrera (Ospedale Molinette, Torino).

Publisher's Note Springer Nature remains neutral with regard to jurisdictional claims in published maps and institutional affiliations. 